\title{
Integración de la documentación legal electrónica a través de LEXML
}

\section{María José Vañó Vañó}

Universidad de Valencia (España)

\section{Resumen}

El intercambio de información legal a través de medios de comunicación a distancia obliga a crear herramientas que eviten la distorsión de los contenidos. El lenguaje XML permite crear sistemas de ordenadores capaces de automatizar partes de la creación de un documento y del proceso de edición (LEXML-LegalXML). Este trabajo tiene por finalidad el origen, la evolución y el estudio de la situación actual del XML en el ámbito legal. De hecho, se revisará la utilización del mismo como herramienta de seguridad, destacando su carácter público (non propietary), y la interoperabilidad entre sistemas legales. Se revisará el modelo europeo, LEXML, y será comparado con el de Estados Unidos, LegalXML; su objetivo común es la creación de un diccionario a partir de esquemas desarrollados por OASIS. Las conclusiones mostrarán la existencia de comunidades, estados o instituciones que están desarrollando los esquemas basados en XML y elaborando estándares similares que progresivamente se irán integrando en un esquema general (RDF).

Palabras clave: Intercambio seguro de información. XML legal. Digitalización de la información. No repudio de la información.

\section{Abstract}

The legal exchange of information through Internet requires tools that avoid the distortion of the contents. Language XML allows the creation of systems of computers able to automate parts of the process of document creation and editing (LEXML-LegalXML). This paper analyzes the origin, the evolution and the current situation of the XML in the legal area. In fact, we analyze this language as a security tool, emphasizing in the non proprietary function, and the interoperability between legal systems. European model, LEXML, will be reviewed and compared with the USA one, LegalXML; their common objective is the creation of a dictionary from schemes developed by OASIS. The conclusions will show that there are different communities, states or institutions that are developing the schemes based on XML and elaborating similar standards that progressively will be integrated in a general scheme (RDF).

Scire. $15: 1$ (en.-jun. 2009) 87-110. ISSN 1135-3716. 
Keywords: Safe interchange of information. Legal XML. Digitalization of the information. No refusing information.

\section{Introducción}

La información legal está siendo objeto de un importante cambio en relación con el soporte utilizado para ello, al sustituirse el papel por el soporte electrónico. Ello conlleva la necesidad de dotar de seguridad a los soportes en los que se encuentra en redes de ordenadores cerradas y en las transmisiones que de la documentación se hagan a través de redes abiertas. Por ello se necesita recurrir a herramientas que contribuyan a proteger la distorsión del contenido de estos documentos electrónicos (Magnusson Sjöberg, 2002). Son numerosas las normas que permiten controlar el contenido de los datos en circulación: desde la Ley Orgánica de Protección de Datos de Carácter Personal, que obliga a que los ficheros automatizados y en soporte papel cumplan determinadas medidas de seguridad, hasta la Ley de Firma Electrónica, según la cual se permite que la firma electrónica reconocida certifique la validez y autenticidad de un documento, o la Ley de la Administración Electrónica, Ley 11/2007, de 22 de junio, de acceso electrónico de los ciudadanos a los servicios públicos, que reconoce la necesidad de la existencia de unos estándares comunes que permitan la interoperabilidad entre las administraciones públicas (1).

Tanto el ámbito económico como el legal han reconocido la necesidad de crear estructuras de datos que permitan intercambiar información. El intercambio de información en un entorno seguro ha encontrado la solución en el lenguaje XML (Extensible Markup Language), el cual se configura como una nueva generación de lenguajes web (Magnusson Sjöberg, 2002).

Este lenguaje ha supuesto una revolución particularmente importante en el ámbito contractual, en el intercambio de información entre las diferentes administraciones públicas y en el intercambio de todo tipo de información en el ámbito de la gestión empresarial. En este último punto debe destacarse la creación de un lenguaje de los estados financieros de las sociedades acuñado como XBRL (eXtensible Bussines Reporting Language, s. f.) y EBR XML (European Business Register $\mathrm{XML}$, s. f.). Pero no podemos olvidar las iniciativas que desde la perspectiva legal se han iniciado tanto en Europa como en Estados Unidos. Así, en el Viejo Continente, países como Holanda están destacando en la elaboración e integración normativa a través del estándar XML; del mismo modo ocurre en Italia, al asumir la Administración Pública las tareas de estandarizar la información. En Estados Unidos debemos resaltar la iniciativa de la Administración de Justicia, que desarrolla el estándar GJXDM (s. f.). Esta posee algunos elementos funcionales comunes con Legal-RDF (2), aunque sigue existiendo un gran abismo entre ambas especificaciones. A saber: Legal-RDF se centra en marcar los estatutos y otros documentos con etiquetas oficiales y con el objetivo de realizar un marcado posterior de la do-

Scire. $15: 1$ (en.-jun. 2009) 87-110. ISSN 1135-3716. 
cumentación contractual, al entender que el mismo modelo de datos podrá ser aplicado a documentos redactados "en prosa". Legal-RDF se centra en recursos semánticos creados de la Web. GJXDM se centra en marcar los documentos con etiquetas elaboradas dentro del sistema de la justicia siguiendo su propio esquema de creación del documento. Por el contrario, Legal-RDF crea ontologías de los términos que se pueden utilizar en documentos "genéricos", mientras que GJXDM crea un concreto esquema de XML para documentos específicos.

\section{Origen y evolución de XML en el ámbito legal}

Las limitaciones evidenciadas por HTML tanto en la descripción del contenido como en la definición de los enlaces originó la creación del lenguaje XML como vía de superación de las mismas (Arellano, Nogales y Martín, 2003; Vañó, 2005). Por ello, la utilización de XML posee dos vertientes diferenciadas: por una parte, el uso en bases de datos relacionales, en particular en el marco del comercio electrónico, con información permanentemente actualizada; por otra, la aplicación a los documentos con información menos estructurada, con carácter discursivo y no tan dinámica. Es el caso de las referencias a textos de jurisprudencia, normas jurídicas y otro tipo de documentación que contiene «parcialmente» elementos de ambas vertientes. Así ocurre con la documentación depositada en el Registro Mercantil (3) por las sociedades mercantiles o la comunicación de la información obligatoria por parte de las sociedades cotizadas a la Comisión Nacional del Mercado de Valores (4), entre otros tipos de documentos.

En España, XML ha sido utilizado sobre todo para la creación de bases de datos de tipo legislativo, compilaciones legislativas y jurisprudenciales, explotadas fundamentalmente por empresas gestoras de esta información, y bases de datos comerciales (5). Desde la perspectiva oficial, el Boletín Oficial del Estado, el portal administraciones públicas, o, en la Comunidad Valenciana, el proyecto AVANTIC (s. f.), LEXNET, así como la Agencia Tributaria y la CNMV, han utilizado este sistema de intercambio de información de bases de datos basadas en el lenguaje XML.

XML es, por supuesto, un factor relacionado con la creación de seguridad. Si bien sus características básicas (Magnusson Sjöberg, 2002) pueden resumirse en que su formato es público (non proprietary); en que puede asegurar la elaboración de documentos válidos y no rechazados por el receptor; en que posee capacidad para la distribución de los documentos, por ejemplo en CD-ROM, on-line, a través de impresión, etcétera; en que contiene directivas legales a través de un vocabulario de marcado, y en que ofrece seguridad a los mensajes electrónicos, debemos añadir las reglas básicas que deben utilizarse para marcar un documento XML (Arellano, Nogales y Martín, 2003).

Si lo que pretendemos es la interoperabilidad entre sistemas legales, lo cierto es que la metodología basada en ontologías es considerada una de las vías más

Scire. 15 : 1 (en.-jun. 2009) 87-110. ISSN 1135-3716. 
prometedoras de cara a conceptualizar el conocimiento, lo que permite una gestión más flexible en la amplia gama de requisitos y opciones metodológicas (Tiscornia, 2007). En este punto debemos definir la ontología como aquel método por el que se pretende capturar términos abstractos cuya estructura principal está formada por taxonomías que describen las relaciones especiales entre los conceptos. El problema que encontramos en el ámbito legal europeo es fundamentalmente la conexión de información multilingüe y de diferentes ámbitos a través de un mapa conceptual que facilite la interoperabilidad.

De hecho, la publicación de la legislación y el desarrollo de herramientas para trabajar con ella es una tarea que requiere la estandarización del XML con el fin de facilitar el intercambio y crear una plataforma para el desarrollo de software legal genérico. Se abre pues, para los creadores de software, un nuevo mercado, y para los consumidores institucionales de XML se resuelve un importante problema: cómo gestionar los diferentes formatos de XML en la misma infraestructura de tecnologías de la información y comunicación. De hecho, el incremento de la convergencia en la Unión Europea a través del aumento de la circulación de personas, servicios, bienes y capital ha ampliado la necesidad de gestionar la legislación proveniente de diferentes fuentes. En la pasada década, los legisladores empezaron a adoptar estándares XML para las fuentes formales, e incluso se ha llevado a cabo una cierta actividad de estandarización a nivel supranacional. El problema es que los estándares están siendo utilizados fundamentalmente por organismos públicos, y la coordinación y cooperación entre organismos nacionales y entre Estados miembros está resultando muy lenta desde la perspectiva de los consumidores por las dificultades técnicas con las que se están encontrando para la estandarización.

El grado de desarrollo de estas superestructuras en Estados Unidos y en Europa en estos momentos es diferente. Las necesidades de seguridad que desde los atentados del 11 de septiembre han surgido en aquel país, así como la homogeneidad en la cultura y en el ordenamiento jurídico, han llevado a promover el desarrollo XML de datos que afectan, fundamentalmente, a la seguridad interior (6). En Europa, por el contrario, los grupos de interesados (7) que iniciaron la vía hacia la estandarización fueron pequeñas comunidades en determinados países que trabajaron en ámbitos muy concretos y que fueron creando nodos en diversos países con el fin de intercambiar y divulgar los trabajos realizados. Así es como surgió la red LEXML.

\section{Modelos de aplicación de XML en el ámbito legal}

\subsection{Modelo europeo. Red LEXML}

\subsubsection{Red LEXML}

En Europa se constituyó en el año 2000 un grupo coordinado por Murk Muller cuyo objetivo era desarrollar un diccionario multilingüe y multijurisdiccional 
para el ámbito legal. El proyecto dio lugar a la creación de comunidades divididas por materias y países que han colaborado en su desarrollo y que han incorporado el método del RDF dictionary como método estándar dentro del ámbito europeo.

La utilización del diccionario RDF para el mundo legal y los avances significativos que ello implicaría en relación con el intercambio de datos ha sido defendido especialmente por John MacClure en Estados Unidos y por Murk Muller en Europa. Ambos creen imprescindible crear una fuente común, o base de datos de elementos, construida a partir de DTD (8) y esquemas XML (9). Dado que Internet es una red global, debe adaptarse a ella un intercambio legal de datos. Por ello en la actualidad se están desarrollando dos proyectos simultáneamente en Estados Unidos y en Europa, dirigidos por los autores citados. Ambos proyectos trabajarán juntos sobre ciertas partes. El objetivo principal de desarrollo será un modelo de datos que será compartido por ambos diccionarios.

La utilización del sistema RDF, o de descripción de sintaxis, es el método más útil para describir esta fuente común y ofrece mayores ventajas que los esquemas XML, más utilizados en Estados Unidos, menos flexibles y con un enfoque exclusivo hacia la Administración de Justicia estadounidense.

LEXML (s. f.) ha sido creado para servir al interés creciente en el intercambio de datos legales. Se trata de un foro abierto para el dominio legal con el fin de compartir ideas y experiencias asociadas a XML. Este foro es el punto de coordinación y de trabajo para el desarrollo de estructuras estandarizadas, vocabularios y herramientas de intercambio de datos. LEXML persigue sus objetivos en concreto a través de la elaboración de un modelo de datos legal y global y del desarrollo de una fuente abierta de un programa oficial legal, que hable y entienda XML.

LEXML es una red de comunidades independientes organizadas las cuales pueden ser jurisdicciones orientadas como Austria, Alemania, Países Bajos, Suecia, España, Italia o por materias. Se trata de una red descentralizada pero que tiende a una aproximación paulatina a través de listas de distribución, asambleas y trabajo conjunto por medio de proyectos internacionales.

La propuesta es la creación de un diccionario legal que contenga todos los posibles elementos, los conceptos necesarios para crear bases de datos legales (10). En el prototipo de diccionario RDF propuesto por Murk Muller (2002) se estructura un XML Schema (11) básico alrededor del concepto de proceso judicial (judgement) en tres idiomas (alemán, holandés e inglés), y ahora también en español.

John MacClure, en Estados Unidos, ha propuesto un diccionario similar al $R D F$ dictionary que contenga todos los términos legales que pudieran aparecer en la DTD XML Legal (12).

La creación de un diccionario para Europa que contenga todos los posibles elementos de las estructuras de datos presentes y futuras podría, por las mismas Scire. 15 : 1 (en.-jun. 2009) 87-110. ISSN 1135-3716. 
razones de diversidad mencionadas antes, ser una tarea casi insuperable. Precisamente porque existen bastantes discordancias en el seno comunitario sobre cuál es el mejor modo de abordar este proyecto, y sectorialmente se están llevando a cabo trabajos paralelos (13): Por ejemplo, la comunicación de información a la CNMV por sociedades cotizadas, Registro Mercantil, Administración Tributaria y Ministerio de Justicia a través de LEXnet.

Desde el punto de vista práctico lo más conveniente es que se formulen propuestas de estandarización desde ámbitos reducidos, áreas geográficas concretas o sectores determinados que confluyan primero en el ámbito nacional para alcanzar el internacional o supranacional en última instancia (Vañó, 2005).

Estos diccionarios deberán complementarse y reforzarán otros formando una red. Cada uno puede aprovechar el trabajo realizado por otros diccionarios, por el mecanismo del namespace (14), proveniente del XML. El diseño del diccionario permitirá un crecimiento orgánico pero no solo desde un determinado diccionario, sino también de la estructura de la red.

\subsubsection{Vinculación de LEXML y desarrollo de RDF-LegalXML}

El grupo de trabajo de LegalXML en Estados Unidos tiene por objeto el desarrollo de un diccionario a partir de las DTD que se están elaborando en el seno de OASIS por iniciativa de John MacClure, el cual contendrá todos los términos legales de lengua inglesa (jmcclure@ hypergrove.com). Se trata de Hypergrove Engineering Inc. (www.hypergrove.com). Este proyecto colabora estrechamente con el dirigido por LEXML. La cooperación a largo plazo ha sido comunicada a través de la declaración hecha pública por John MacClure y Murk Muller (http://www.lexml.de/statement_rdf.htm).

En la actualidad, el vínculo permanece a través de LEXMLWiki, en cuyo espacio se integran todos los modelos elaborados y que formarán parte de Legal-RDF (s. f.). La integración de Legal-RDf en la página Wiki es progresiva y está todavía incompleta. En la actualidad se cuenta con una segunda versión, con algunos cambios significativos que persiguen conseguir un modelo de programación de buenas prácticas que permita desarrollar herramientas de software a partir de la ontología.

El modelo de datos Legal-RDF describe en abstracto el modo en que el dato es representado en un sistema de información o sistema de gestión de bases de datos. Es esencial el contexto, que está en estos momentos en fase de desarrollo. Así, por ejemplo, el actors model define e incluye los derechos de las personas y organizaciones que tienen un cierto grado de reconocimiento legal. En este modelo, el principal objetivo es establecer los patrones del diseño orientados por técnicas de programación.

En el caso del modelo de documento, las clases que se definen en él relacionan la estructura y la presentación de un documento típico, con el fin de lograr menos 
pérdidas en las transformaciones. Entre los modelos nos interesa destacar el llamado dramas model, relativo a la definición de los hechos que pueden ocurrir y afectar a las circunstancias recogidas en el resto del documento. De hecho, en él se incluirá como última clase, base del modelo, el EventContext, siendo el ActionContext y el ActivityContext subclases. El modelo económico introduce definiciones integradas de 15 áreas diferentes, relativas a capital, trabajo y transacciones materiales. El modelo de derecho y política (law and politics model) contiene definiciones de normas, regulaciones u otros mecanismos legales de sistemas políticos necesarios para la creación de normas jurídicas. Derechos, garantías, etcétera, son definidos en él. Ninguna de las fuentes en este modelo es válida como contexto independiente.

El modelo semántico Legal-RDF incorpora el estándar Dublín Core Attributes (15) junto con Directed Acyclic Grapas (16). El objetivo principal es considerar un conjunto de elementos como un único texto que permita a su vez identificar cada una de las partes con el fin de facilitar información y vincularla con enlaces, internos y externos, que puedan enriquecer el contenido del texto principal. Estamos hablando por tanto de un texto extensible (17). Las ontologías definidas para el texto deben ser capaces de relacionar objetos o propiedades de texto con las colecciones de una clase determinada, vinculadas al mismo tiempo con el texto principal. Y, a su vez, estas referencias deberán aparecer como definición para otras propiedades.

\subsubsection{Iniciativas institucionales europeas}

Las iniciativas europeas en el marco de la utilización del lenguaje XML han sido fundamentalmente orientadas en el ámbito institucional hacia la interoperabilidad de sistemas, contenidos y procesos entre los diferentes sujetos de la Administración, desde la local hasta la europea. Ello se ha propiciado desde las instancias europeas con el fin de mejorar sustancialmente las relaciones con los organismos públicos en todos los niveles. En particular, la Decisión 2004/387/CE del Parlamento Europeo y del Consejo de 21 de abril de 2004 relativa a la prestación interoperable de servicios paneuropeos de Administración Electrónica al sector público, las empresas y los ciudadanos (IDABC) constituye la base legal del programa IDABC para el período 2005-2009.

Las ventajas proporcionadas por el citado plan para la puesta en marcha de un marco común que permita intercambiar y compartir datos e información, reducir los trámites administrativos, limitar la burocracia y ahorrar tiempo de un modo sustancial ha facilitado una serie de medidas horizontales entre las cuales podemos enumerar las siguientes:

- Desarrollo de una aplicación basada en una especificación de vocabularios XML, esquemas y entregables XML relacionados con el apoyo al intercambio de datos en redes.

Scire. 15 : 1 (en.-jun. 2009) 87-110. ISSN 1135-3716. 
- Requisitos de modelos funcionales y no funcionales para la gestión de registros electrónicos en las administraciones públicas.

- Un marco de metadatos para aplicaciones paneuropeas de la información del sector público.

- Comparación de normas abiertas de intercambio con miras a crear una política en formatos abiertos (18).

Este plan, junto con el plan de acción i2010 (19), prevé como complemento del primer plan de acción para la interoperabilidad europea el desarrollo de aplicaciones y la necesidad de armonizar contenidos, seleccionar y adoptar servicios genéricos y utilizar la infraestructura de redes telemáticas.

Por otra parte, la Decisión 2004/387/CE enumera de forma pormenorizada en su anexo II los ámbitos de las medidas horizontales en apoyo de los Proyectos de Interés Común. En relación con los servicios de infraestructuras fija la siguiente relación, prestando una especial atención al apoyo a la creación de estándares y vocabularios basados en XML:

- Plataforma de comunicación segura y fiable para intercambio de datos entre AA. PP.

- Sistema seguro y fiable para gestión de flujos de datos.

- Juego común de herramientas para la gestión de sitios y portales web plurilingües.

- Acreditación de plataforma a fin de manejar información clasificada.

- Creación y aplicación de una política de autenticación.

- Estudios de seguridad y análisis de riesgo en apoyo de redes u otros servicios.

- Mecanismos que establezcan la confianza entre las autoridades de certificación.

- Servicios de autorización, autenticación para los proyectos de interés común.

- Marco común para compartir e intercambiar datos e información.

- Especificación de vocabularios XML, esquemas y entregables.

- Requisitos para la gestión de registros electrónicos en AA. PP.

- Marco de metadatos para aplicaciones paneuropeas.

- Comparación de normas abiertas de intercambio para política en formatos abiertos.

- Especificaciones comunes y servicios que faciliten la contratación pública electrónica.

- Sistemas de traducción automática y otras herramientas plurilingües.

- Aplicaciones en apoyo al trabajo de cooperación entre administraciones públicas.

- Aplicaciones en apoyo al acceso multicanal a los servicios.

- Herramientas basadas en programas de fuente abierta y acciones que faciliten intercambio de experiencias entre AA. PP.

Además, en la Recomendación 11, técnica, indica expresamente que deben utilizarse estándares basados en XML junto a otras medidas de esta naturaleza.

Por otra parte, el IDABC se desarrolla a través del plan de acción i2010, por el que se afianza la interoperabilidad, desplegando la hoja de ruta de desarrollo de la contratación electrónica y abriendo servicios de alto impacto para los ciudadanos (20) y para las empresas (21), lo que se lleva a cabo a través de varios portales y herramientas como Your Europe (s. f.) y TESTA (Trans European Services

Scire. $15: 1$ (en.-jun. 2009) 87-110. ISSN 1135-3716. 
for Telematics between Administrations) con la implantación de servicios que permitan la interoperabilidad de las administraciones públicas en Europa (22).

\subsubsection{Estado actual en la Administración Pública española}

En nuestro país, como hemos indicado en la introducción de este trabajo, con la promulgación de la Ley 11/2007 de acceso electrónico de los ciudadanos a los servicios públicos se convierte el derecho de los ciudadanos a relacionarse con las administraciones públicas de forma electrónica en servicio universal, cuyo plazo para hacerse efectivo será el 31 de diciembre de 2009.

A partir de esta fecha, todos los trámites de carácter público deberán ser accesibles electrónicamente; en la Ley se regulan las condiciones para hacer efectivo este proyecto y se prevé la cobertura del vacío legislativo existente en algunos aspectos de las relaciones entre ciudadanos y administraciones públicas, favoreciendo y garantizando la interoperabilidad de las soluciones tecnológicas desarrolladas por las distintas administraciones.

En el marco de la interoperabilidad de las administraciones públicas, el título cuarto, relativo a la cooperación entre administraciones para el impulso de la Administración Electrónica, se trata el marco institucional de cooperación en dicha materia, contemplando de manera específica la seguridad, los sistemas y las soluciones para favorecer la interoperabilidad (23).

Se fomenta la cooperación entre administraciones a través de la prestación conjunta de servicios a los ciudadanos (art. 4) y se promueve el principio de neutralidad tecnológica y de adaptabilidad al progreso de las técnicas y sistemas de comunicaciones electrónicas, garantizando la independencia en la elección de las alternativas tecnológicas por los ciudadanos y por las administraciones públicas. Para ello se utilizan estándares abiertos, así como, en su caso, estándares que sean de uso generalizado por los ciudadanos. A efectos de controlar y asegurar la compatibilidad e interoperabilidad entre los sistemas y aplicaciones utilizadas por las administraciones públicas, el artículo 40 configura estas funciones para el Comité Sectorial de la Administración Electrónica.

El artículo 42 de la Ley establece que el Esquema Nacional de Interoperabilidad deberá comprender el conjunto de criterios y recomendaciones de seguridad, conservación y normalización de la información, de los formatos y de las aplicaciones que deberán ser tenidos en cuenta por las administraciones públicas para la toma de decisiones tecnológicas que faciliten la interoperabilidad. Así, señala expresamente:

1. El Esquema Nacional de Interoperabilidad comprenderá el conjunto de criterios y recomendaciones en materia de seguridad, conservación y normalización de la información, de los formatos y de las aplicaciones que deberán ser tenidos en cuenta por las Administraciones Públicas para la toma de decisiones tecnológicas que garanticen la interoperabilidad.

Scire. $15: 1$ (en.-jun. 2009) 87-110. ISSN 1135-3716. 
2. El Esquema Nacional de Seguridad tiene por objeto establecer la política de seguridad en la utilización de medios electrónicos en el ámbito de la presente Ley, y está constituido por los principios básicos y requisitos mínimos que permitan una protección adecuada de la información.

3. Ambos Esquemas se elaborarán con la participación de todas las Administraciones y se aprobarán por Real Decreto del Gobierno, a propuesta de la Conferencia Sectorial de Administración Pública y previo informe de la Comisión Nacional de Administración Local, debiendo mantenerse actualizados de manera permanente.

4. En la elaboración de ambos Esquemas se tendrán en cuenta las recomendaciones de la Unión Europea, la situación tecnológica de las diferentes Administraciones Públicas, así como los servicios electrónicos ya existentes. A estos efectos considerarán la utilización de estándares abiertos así como, en su caso y de forma complementaria, estándares que sean de uso generalizado por los ciudadanos.

\subsubsection{Aplicación de XML: MetaLex, Norme in Rete, LOIS}

Los proyectos más significativos vigentes en la actualidad y vinculados en su origen a la red LEXML son fundamentalmente el proyecto holandés MetaLex y el italiano, a través de Norme in Rete y LOIS. Sin embargo, deben destacarse otros, como el proyecto LexDania de Dinamarca, LexML de Brasil, E-Lex de Austria o CHLex en Suiza.

\subsubsection{Premisas y desarrollo del proyecto MetaLex en Holanda}

En el seno del proyecto E-Power (24) y E-Court (25) se anunció en septiembre 2002 el lanzamiento de MetaLex con un formato abierto y una estructura genérica y extensible para codificar la estructura XML y RDF de documentos legales (Boer y Winkels, 2007; Boer, 2007). La versión actual del esquema MetaLex (s. f.) incorpora las buenas prácticas de diseño del proyecto italiano Norme in Rete y el estándar panafricano AKOMA NTOSO XML (26). Del mismo modo, en el marco de MetaLex se ha desarrollado la especificación legal LKIF (Legal Knowledge Interchange Format) (27) y aplicado al proyecto ESTRELLA (European Project for Standardized Transparent Representations in order to Extend Legal Accessibility, s. f.) de la Unión Europea.

Los elementos básicos destacados en MetaLex son la creación de un estándar nacional compatible con CEN MetaLex, la utilización sistemática de los estándares W3C, la separación de la estructura, los contenidos normativos, la presentación y los metadatos y la posibilidad de excepciones, extensiones y convertidores.

Un estándar de estas características, abierto, permite facilitar y llevar a cabo una distribución de contenidos legislativos, conservar a largo plazo las inversiones y soportes que permitan la integración de contenidos y comparar resultados. Pero lo importante ya no es tanto que se adapten a estándares relevantes, sino que puedan inventar su propio estándar, que permita facilitar la interoperabilidad internacional. El conjunto de documentos que pueden visualizarse a través de CEN 
MetaLex son sentencias, contratos, jurisprudencia, actas y procedimientos parlamentarios (28).

El principal objetivo de MetaLex era desarrollar una ontología como definición de términos utilizados para describir y representar un área de conocimiento. Como señala Winkels, la ontología responde a las preguntas ¿qué es? y ¿qué sabemos?

Lo que se persigue con las ontologías es capturar los términos más abstractos posibles en el ámbito de la práctica; por ello la ontología se define en el ámbito de la informática como un intento de formular un exhaustivo y riguroso esquema conceptual dentro de un dominio dado, con el fin de facilitar la comunicación y el intercambio de la información entre diferentes sistemas (Breuker y Winkels, 2007).

La estructura básica de la ontología es un cuerpo principal denominado taxonomía y unas propiedades que deben ser necesarias y suficientes. Los niveles semánticos incorporan en la base los denominados diccionarios: en el primer nivel, taxonomías que describen las relaciones especiales entre los conceptos; en el segundo nivel se incorpora el llamado tesauro, donde se añadirán a las taxonomías relaciones léxicas —-sinónimos, hiperónimos o partonomy (29)—; en el tercer nivel se situarán los modelos de referencia, combinando algunas relaciones anteriores y otras axiomáticas.

Mientras que MetaLex es una tecnología que permite el intercambio de sistemas basados en conocimiento legal, entre otros usos, LKIF trata también de la capacidad de intercambio de la representación del conocimiento legal. La interpretación de la ley es muy difícil de estandarizar a través de XML, pero tiene un gran potencial en el mercado. La representación del conocimiento legal es, o debería ser, una actividad continua de las administraciones públicas, porque se deberían adoptar los cambios de legislación e interpretación siguiendo la jurisprudencia, los cambios en la política... En ausencia de estándares, habría de aceptarse el LKIF (30).

Lo que se pretende a partir de MetaLex es procurar la interacción, la interoperabilidad entre la legislación para evitar que se incremente la complejidad de la internacionalización de normas. A través de las tecnologías de la información y comunicación, junto con la elaboración de estándares, se conseguirá un sistema legal más efectivo y eficiente. Pero para ello se necesita elaborar elementos comunes a todos los recursos o fuentes legales y crear compilaciones de elementos sencillas que permitan que todos los documentos legales puedan intercambiar sus datos. Así, lo que se debe estandarizar es la estructura del documento, las referencias, la identidad, los metadatos y los vínculos a la representación de contenidos.

La herramienta que permite desarrollar este aspecto es MetaVex. Las condiciones o requerimientos mínimos son que exista independencia en la jurisdicción, también en el uso del lenguaje, que sea extensible de manera que facilite su lectura e interpretación, que se utilicen los estándares W3C y que se integre en software libre.

Scire. 15 : 1 (en.-jun. 2009) 87-110. ISSN 1135-3716. 
Los problemas que se plantean en las respectivas jurisdicciones son, por una parte, los organismos, cuyo vocabulario y principios constitucionales pueden ser diferentes, aunque es posible que tengan similitudes en cuanto a la forma (propuesta, adopción, firma, publicación, entrada en vigor, contratos, legislación de consumidores, jurisprudencia...). Pero además de estas similitudes podemos encontrar diferencias relativas a la fundamentación, o bien en los detalles de procedimiento, o distinciones entre realidad y realidad institucional de jure y de facto. A tales efectos, el legislador holandés ha preparado unos estándares para elaborar sus propias normas, pero pueden modificarse con el tiempo. Mucha doctrina aparece también implícita en numerosas instituciones, y en algunas de una manera explícita.

MetaLex distingue entre diferentes partes del documento, así como las referencias en su interior y a otras fuentes y conceptos legales. Las versiones legales son consideradas fuentes orgánicas legales publicadas por las autoridades correspondientes. El poder legal, legislador o poder superior del que emanan las normas, la región o ámbito geográfico al cual se debe aplicar la fuente, y la diferenciación entre texto primario, exposición de motivos y tercera parte anotada, y los metadados o datos relacionados con otros también son etiquetados.

Los principales elementos contenidos en un documento son Article, como elemento estructural con una única identificación en el nivel artículo del documento; CitationDesignation, que es la plena identificación del documento que se usa en citas o referencias; IndexDesignation, que identifica los elementos estructurales y su utilización en las citas, categorías e índices; List, frase completa o fragmento de una frase contenida en un listado que está estructurado e indexado en forma de lista vertical; Part, un elemento estructural cuya identificación única contiene una parte o artículos de un texto; Sentence, una unidad estructural mínima de un documento, que es una frase completa y no contiene otras marcas de unidades estructurales; SentenceFragment, estructura mínima de un documento, que es una parte de una frase completa; SentenceFrangmentSubpart, una subparte indexada de una lista; SubPart, elemento estructural con una única identificación contenida en un artículo o subparte; Title, identificación de un elemento estructural; Cite, referencia sistemática textual; y Reference, referencia textual no sistemática.

\subsubsection{Movimiento LEXML en Italia: Norme in Rete, LOIS}

En cuanto al movimiento LEXML (s. f.) en Italia, fue iniciado por Monica Palmirani y seguido muy de cerca inmediatamente por la Administración Pública, que incorporó los modelos y estándares elaborados. En ese país, la utilización de XML en el ámbito legal vino motivada por la necesidad de archivar los documentos jurídicos y transferir la información teniendo en cuenta el valor estructural y siempre semántico de las partes del texto. El intercambio de información es esencial en el seno de los poderes públicos, y se realiza de modo horizontal y vertical. Por ello 
se ha propiciado un proyecto con respaldo institucional a través del cual se desarrollan diversas líneas de trabajo. Se pretende, por ejemplo, que la doctrina jurídica publicada en diarios oficiales tenga el soporte de XML, para lo que se creó un software denominado lexedit como soporte para los textos legislativos (31).

En el Ministerio de Justicia se ha creado un portal de gestión de servicios y creación de software para el estándar, Norme in Rete (NIR), es decir, Normas en la Red. También, desde 2002, el Instituto Superior para la Prevención y Seguridad en el Trabajo ha preparado varias iniciativas en este ámbito (32).

El objeto de LOIS (Lexical Ontologies for Legal Information Society) es crear un paradigma para tener acceso a recursos legales desde un punto de vista lingüístico monolingüe y cruzado. Uno de los obstáculos con que se encuentra un ciudadano es la jerga legal. De hecho, varios conceptos legales pueden tener diversas denominaciones o definiciones en el lenguaje común y en el legal. Por ello se ha creado una relación entre diferentes estándares europeos, relacionados o correspondientes al lenguaje estándar, de manera que la localización de los recursos proporcione una vía formal de mapa de lenguaje estándar correspondiente con jerga legal y viceversa. Así pues, cualquier usuario podrá realizar búsquedas en LOIS utilizando terminología de uso general, recibiendo los correspondientes documentos con las traducciones técnicas. Igualmente para los usuarios especializados que podrían tener problemas en la recuperación de cierta clase de documentos empleando las palabras clave apropiadas (33) se está utilizando el mismo sistema, incluso para decisiones judiciales donde el estilo argumentativo está en muchas ocasiones afectado por la ambigüedad; también se pueden beneficiar de esta interconexión entre diferentes estados europeos con diferentes lenguajes.

Por otra parte, el programa Norme in Rete es un punto de acceso a la normativa italiana y europea publicada sobre la Web. Se trata de fomentar y difundir el patrimonio informativo común. Se ha conseguido con ello el acceso gratuito a todas las normas a través de Internet desde 1999, extendido a toda la normativa estatal desde 1904 y a la regional desde 1948. Se establece un estándar de marcado e identificación de la documentación basada en XML y URN (Uniform Resource Name) fomentando la interoperabilidad entre los diferentes sistemas informáticos y automatizando el proceso interno. Se ofrece formación on-line a través de cursos dedicados a los operadores sobre los esquemas DTD y el lenguaje XML. Norme in Rete no es solo un motor de búsqueda de normativa, sino que está integrado en el estudio y evolución del estándar para la representación y gestión de la documentación legislativa en el ámbito nacional e internacional.

De hecho, se está multiplicando la representación del estándar italiano en otros estados adaptándolo a cada uno de ellos. Este programa ha sido recogido a través de la Ley de 23 de diciembre de 2000, número 388. 
3.1.4.3. Interconexión e interoperabilidad entre los registros mercantiles europeos

Una especial atención debe prestarse a la iniciativa de los registradores mercantiles europeos para fomentar la interoperabilidad de los registros mercantiles, impuesta por la modificación de la Primera Directiva de Sociedades. Esta propuesta es importante no ya por su consideración de bases de datos interconectadas, cuyo acceso es público, sino como sistema de control de la gestión empresarial a efectos de generar transparencia, y donde los sujetos interesados no son solo los registros mercantiles, sino también la Comisión Nacional del Mercado de Valores, el Banco de España, la Agencia Tributaria, socios e inversores.

Esta iniciativa ha sido desarrollada como consecuencia de la modificación de la Primera Directiva de Sociedades, en la que se centraliza la publicidad de los registros mercantiles y se pretende fomentar la interconexión entre los registros mercantiles de los diferentes Estados miembros. Concretamente, la modificación de la Primera Directiva sobre sociedades del día 11 de junio de 2003 obliga a incorporar una tecnología que permita el intercambio electrónico de información y que sea a la vez capaz de ofrecer seguridad a los usuarios de dicha información (34). Este lenguaje no es otro que el XML (35).

En su origen se elaboró el estándar crXML (commercial register XML), con lo que se ha convertido en el proyecto BRITE (Business Registers Interoperability Throughout Europe).

Los organismos asociados (36) a este proyecto fueron el Registro Mercantil Europeo (37), el Norway Register Development (38) y el propio Foro de los Registradores Mercantiles Europeos (39).

Aunque es posible utilizar otro sistema para la aplicación crXML a la información depositada en los registros (2001, $\$ 639$ a 656), se buscaba que estos adoptaran directamente este estándar de manera que el intercambio se hiciera directamente en crXML. En ese caso sería útil que los servidores locales dispusieran de esquemas relativos a la especificación, así como de una lista de códigos.

La versión actual de la interoperabilidad ha sido denominada proyecto BRITE. Sus objetivos son fundamentalmente establecer un marco que permita la interoperabilidad de los registros mercantiles en Europa. Del mismo modo, transforma y armoniza procesos administrativos aislados en acciones cooperativas y coordinadas. También añade transparencia a varios perfiles de negocio y mejora el acceso público a los documentos archivados.

De manera específica, BRITE persigue eliminar barreras administrativas con el fin de consolidar la libertad de establecimiento y de movimiento de las sociedades. También busca el desarrollo y la adopción de sistemas de soporte adaptables y proactivos en el e-Government. Se trata de lograr una actitud proactiva y positiva al cambio, innovación y previsión de servicios en los organismos públicos; avan- 
zar en el estado del arte en los procesos de diseño de los procesos administrativos utilizando ontologías y webs semánticas. Este proyecto permitirá mostrar los datos e indicadores del impacto de los cambios de legislación en los procesos públicos internacionales y en los servicios públicos. Y, por supuesto, desarrollar estándares directamente relacionados con las tecnologías de la información y la legislación societaria, así como facilitar la transferencia entre administraciones públicas.

\subsection{El modelo estadounidense}

En Estados Unidos la interoperabilidad entre la Administración Pública se integra en el NIEM, modelo nacional de intercambio de información. Este proyecto se inició el 28 de febrero de 2005 a través del Departamento de Seguridad Interior, con el fin de desarrollar el marco nacional para el intercambio de datos, y del Ministerio de Justicia. En él se fijaban varios niveles en un modelo de referencia del modelo de datos GJXDM, en el que se incorporan una base, listas de código, dominios, esquemas, hojas de estilo, perfiles y un registro acumulativo de los cambios con el fin de conseguir el mejor intercambio de información en la Administración Pública.

El NIEM es un modelo de referencia, no un estándar rígido, y debe ser utilizado exactamente tal y como es. Fue designado como fuente de una colección de elementos utilizados como líneas base para la creación de documentos de intercambio y transacciones entre la Administración. También existe una representación del esquema XML del modelo entero, aunque no es un requisito esencial para validar el documento. No hay varios requerimientos para que el documento se considere bien formado.

El objetivo del estándar NIEM es lograr que tanto el remitente como el receptor de la información puedan entender del mismo modo, y con un significado inequívoco, esta información. Se pretende asegurar que la fuente básica del conjunto de información (los componentes NIEM) es comprensible y posee el mismo significado en ámbitos diferentes. El resultado permite un nivel de interoperabilidad que sería inalcanzable en caso de que proliferaran esquemas y diccionarios a petición de interesados concretos. Esto es en realidad lo que ocurre en la Unión Europea, pretendiéndose su corrección a partir del programa IDABC.

Podemos reducir los principios NIEM a los siguientes: $a$ ) se deben utilizar los esquemas de referencia de NIEM, válidos en sí mismos, basados en el NIEM Schema namespace; $b$ ) se ha de ser semánticamente consecuente, emplear los elementos NIEM de acuerdo con sus definiciones y no utilizar la excepción de un elemento NIEM.

El modelo formulado por el Ministerio de Justicia de los Estados Unidos (DOJ), por los programas de justicia (OJP), junto con la iniciativa global en este mismo ámbito, ha sido presentado oficialmente a la comunidad de la justicia a través del

Scire. $15: 1$ (en.-jun. 2009) 87-110. ISSN 1135-3716. 
estándar Global JXDM, versión 3.0.3. Con este lanzamiento se consigue aumentar la seguridad de la justicia y del público en relación con la información ofrecida facilitando la interoperabilidad.

El Global JXDM es un estándar que incluye un modelo y un esquema de XML que contiene a su vez un modelo de los datos, un diccionario de estos y un esquema de XML. A este modelo se le irán añadiendo las adiciones, cancelaciones y modificaciones que puedan aprobarse de manera acumulativa y que se anunciarán en cada publicación del modelo.

El Global JXDM es un estándar de XML diseñado específicamente para los intercambios de información en la justicia, facilitando la aplicación de la ley, su utilización por las agencias públicas de seguridad, los litigantes y los defensores públicos; es una herramienta para compartir con eficacia datos e información de una manera oportuna.

Este estándar utiliza un vocabulario común, permite el acceso de fuentes múltiples y la reutilización en usos múltiples, actualizaciones específicas, referidos al registro global del cambio de JXDM. Una característica importante del Global JXDM, versión 3.0.3, es su compatibilidad con todos los estándares XML elaborados para Global JXDM con versiones anteriores.

El Global JXDM es un modelo de referencia no rígido que debe ser utilizado siguiendo el conjunto de fuentes diseñadas como fuente de intercambio de documentos y transacciones en el ámbito de la justicia. Los principios en los que se basa el estándar son los mismos que en el caso del NIEM. Junto al GJXDM, creado como diccionario completo para integrar las necesidades crecientes de los usuarios, se ha detectado que en la mayor parte de los casos solo se utiliza una parte del estándar global JXDM, por lo que se ha proporcionado un esquema con un conjunto reducido de elementos, de manera que se podrá construir, manualmente, un subesquema.

\section{XBRL: un estándar para el intercambio de información financiera}

La Ley 26/2003 de Transparencia de las Sociedades Mercantiles pretende transmitir al mercado toda la información relevante para los inversores con unas condiciones específicas; a saber: que sea correcta, veraz y que se transmita de forma simétrica equitativa y en tiempo útil. La mejor herramienta que podrá contribuir a cumplir estos requerimientos es la utilización del lenguaje XML, que en el ámbito financiero se ha definido como XBRL, tal y como hemos señalado al comienzo de este breve trabajo (Milani y Mondorf, 2007).

Cierto es que Internet es el marco que permite el cumplimiento de esta premisa de facilitar toda la información; sin embargo, también es cierto que la información que aparece en Internet no está estructurada. La información económica ha 
circulado siempre, pero la necesidad de transparencia de las sociedades cotizadas para con los mercados financieros, motivada por los escándalos de algunas de estas grandes empresas, obligó a que los contenidos mínimos a divulgar se incorporaran a la normativa legal, como por ejemplo la obligación de que la información a los accionistas esté a menos de tres clics de navegación desde la página principal (Circular 1/2004 de la CNMV). En España se hizo a través de la Ley 26/2003 y reglamentos de desarrollo (40). Además, ha sido necesario que entes públicos y privados interesados en estructurar la información relativa a las sociedades cotizadas aúnen esfuerzos con el fin de desarrollar y estandarizar contenidos (Vañó, 2004).

En este marco de necesidad, el lenguaje informático XML ofrece numerosas posibilidades de concreción. Ya en 1998, Charles Hoffman, experto contable y auditor, utilizó XML con el fin de simplificar la automatización del intercambio de información financiera. En pocos años consiguió atraer el interés primero y el respaldo después de un conjunto de individuos y organismos públicos y privados. Estos interesados se agruparon en numerosos países a través de la organización XBRL Internacional (Vañó, 2005). En España, la Asociación Española de Contabilidad y Administración de Empresas (AECA) propuso en febrero de 2002 a XBRL Internacional que España se constituyera en jurisdicción a través de la Comisión de Nuevas Tecnologías y Contabilidad de AECA. En el seno de esta comisión se creó un grupo de trabajo para el desarrollo de XBRL. Los miembros fundadores fueron la Central de Balances del Banco de España, Informa, S. A., el Instituto de Auditores Censores Jurados de Cuentas de España, Ambición (hoy Microsoft Business Solutions), PricewaterhouseCoopers y la Universidad de Huelva.

En marzo de 2004 se confirmó como jurisdicción definitiva, siendo el Banco de España la principal institución interesada en incorporar XBRL y quien ostenta la presidencia de XBRL España; en la vicepresidencia se sitúa la persona que introdujo XBRL en España, Enrique Bonsón.

En la actualidad existen varios subgrupos que están llevando a cabo desarrollos de esquemas XML o taxonomías: DGI (Datos Generales de Identificación), IPP (Información Pública Periódica de empresas con valores admitidos a cotización) y PGC90 (Plan General de Contabilidad de 1990, Plan General Contable para el sector público). Recientemente el Banco de España ha iniciado un proyecto cuyo objetivo es el desarrollo de un sistema de intercambio de información financiera (SIIF). Entre los agentes que participan se encuentran las entidades de crédito. La nueva normativa internacional, que obliga a adoptar las normas IAS/IFRS (Bonsón, Coffin y Watson, 2002; Bonsón y Andrés, 2003, pp. 35 y ss.) a partir de 2005 a los grupos consolidados que coticen en mercados secundarios oficiales, y que ha modificado la Circular 4/1991, permitirá la utilización del estándar XBRL para soportar el intercambio de información entre el Banco de España y las entidades de crédito sometidas a su supervisión y control (41).

Scire. $15: 1$ (en.-jun. 2009) 87-110. ISSN 1135-3716. 
En nuestro país, la Ley sobre Transparencia de las Sociedades Cotizadas obliga a difundir a través de las páginas web determinados contenidos y atribuye la función de supervisión y control a la Comisión Nacional del Mercado de Valores. Este organismo supervisor, al igual que el Banco de España, está muy interesado en participar y colaborar en las tareas desarrolladas por XBRL, dado que el intercambio de información de acuerdo con este estándar permitirá controlar contenidos y estructuras. La elaboración de formularios de acuerdo con unos contenidos que permitan su validación por sí ahorrará costes a la CNMV. En realidad, así será posible llevar a cabo una auditoría continua de la información garantizando a los inversores que los datos divulgados por la CNMV y por las webs corporativas son exactamente los mismos (42).

Al hecho de contrastar la información publicada en la página web con la información oficial depositada en los correspondientes registros públicos y el respeto a la normativa sobre transparencia está contribuyendo el Colegio de Registradores de la Propiedad y Mercantiles de España al crear un sello de calidad que se incorpora en aquellas páginas web que ajusten su contenido a la normativa que sobre transparencia ha elaborado nuestro Legislador (43). El usuario podrá acceder al contenido de la página web de la sociedad cotizada con la máxima garantía de fiabilidad y autenticidad.

Del mismo modo surgió el interés por parte de los registradores mercantiles europeos, que, a la vista de la modificación de la Primera Directiva de Sociedades (44) y la propuesta de creación de un espacio registral único europeo, consideraron necesario desarrollar un estándar para el intercambio de datos entre registros y otras autoridades públicas. El estándar crXML ha sido utilizado en gran medida en el subgrupo de XBRL DGI (Datos Generales de Identificación), que ha publicado el borrador del esquema en marzo de 2005 (45). El estándar DGI de XBRL España tiene por objeto la transmisión electrónica de los datos registrales de entidades e individuos y de las estructuras de información general asociadas a los mismos, así como de información de interés general, de acuerdo a diversas instituciones oficiales.

\section{Notas}

(1) Art. 25.4: «Podrán aportarse documentos que acompañen a la correspondiente solicitud, escrito o comunicación, siempre que cumplan los estándares de formato y requisitos de seguridad que se determinen en los Esquemas Nacionales de Interoperabilidad y de Seguridad. Los registros electrónicos generarán recibos acreditativos de la entrega de estos documentos que garanticen la integridad y el no repudio de los documentos aportados».

(2) RDF, o Resource Description Framework, es otro lenguaje XML que incluye información sobre la página en la que ha sido definido. Esta información, también llamada metadatos, indica a los buscadores o demás aplicaciones que hagan uso de las características de la información que se encuentra contenida en esta página o servidor. Es algo similar a cualquier documento XML válido. Los documentos RDF constan de diver-

Scire. $15: 1$ (en.-jun. 2009) 87-110. ISSN 1135-3716. 
sos nodos, dentro de cada uno de los cuales se incluyen una serie de atributos sobre los que se definen los valores que identifican a ese servidor frente a los demás. Los servidores, a su vez, están definidos por la página web. Por tanto, la ventaja del RDF es que la descripción de los recursos web se consigue a través de la referencia a otros recursos, lo que no es posible en un sistema cerrado. Vid. http://www.w3.org/RDF (200710-12).

(3) Vid. al respecto el proyecto de interoperabilidad de los registros mercantiles europeos en http://www.briteproject.net (2007-10-12).

(4) Se puede consultar la documentación relativa a la comunicación de información obligatoria en http://www.cnmv.es/XBRL/InformesXBRL.htm (2007-10-12). Sobre el papel de XBRL como específico para el sector financiero, vid. Bonsón, Coffin y Watson (2002); Bonsón y Andrés (2003).

(5) Vid., por ejemplo, Derecho.com (www.derecho.com), o bien Tirant on Line (www. tirantonline.com) o www.lexnova.com, o, como web institucional, Eur-Lex como servicio de publicaciones de la Unión Europea (http://eur-lex.europa.eu/es) (2007-10-12).

(6) Vid. http://it.ojp.gov/jxdm (2007-10-12).

(7) Vid. www.uv.es/lexml (2007-10-12).

(8) Definición del tipo de documento. Su función básica es la descripción del formato de datos, para usar un formato común y mantener la consistencia entre todos los documentos que utilicen la misma DTD. De esta forma, dichos documentos pueden ser validados, conocen la estructura de los elementos y la descripción de los datos que trae consigo cada documento y pueden, además, compartir la misma descripción y forma de validación dentro de un grupo de trabajo que usa el mismo tipo de información. http://es.wikipedia.org/wiki/DTD (2007-07-18).

(9) Vid. la declaración realizada por Murk Muller y John MacClure en Berlín, en mayo de 2001, en http://www.lexml.de/statement_rdf.htm (2007-07-18).

(10) Así por ejemplo, el diccionario RDF creado por la empresa Dataconsortium ha mostrado que es posible crear una DTD desde el diccionario RDF con un simple clic de ratón. El cuerpo de elementos de este diccionario tiene actualmente un nivel superior de 15 términos base, que pueden convertirse en 5 en el futuro. Un segundo nivel lo ocupan aproximadamente 109 términos, que son categorías de cada término base. Los restantes 9000 términos provienen de la primera y la segunda capa no de modo jerárquico, sino en la forma de red de términos vinculados con la característica de multiculturalidades del lenguaje RDF.

(11) No debemos olvidar que los XML Schema son documentos XML que describen la sintaxis de otros documentos XML. Por ello, su estructura se basa en dos partes principales: el encabezado XML y el cuerpo. Es precisamente en este último donde se introducen los cambios al incorporar al elemento raíz una serie de atributos que definen en sí al XML Schema. Son básicamente dos: por una parte, el que se encarga de definir la URI o lugar donde se encuentra situado el XML Schema (targetNS); por otra, la versión, que indica el número de la versión del XML Schema que estamos utilizando.

(12) Pueden verse los avances del proyecto en http://www.hypergrove.com/legalxhtml.org/ LegalXHTMLNarrative.html (2007-10-25).

Scire. $15: 1$ (en.-jun. 2009) 87-110. ISSN 1135-3716. 
(13) Sirva como ejemplo la estandarización que están llevando los registradores mercantiles europeos a través del proyecto BRITE (www.briteproject.net) (2007-10-25).

(14) Con este mecanismo se persigue evitar colisiones entre conceptos. Concretamente, mediante el sistema URI (Uniform Resource Identifier) se define un único segmento para un concepto que de forma lógica ofrezca la localización de los recursos de red. Vid. Dick (2000, p. 165).

(15) www.dublincore.org (2007-10-25). De hecho, es una iniciativa llevada a cabo por esta organización, cuyo objetivo es la promoción y difusión de normas interoperables sobre metadatos y el desarrollo de vocabularios especializados en metadatos para la descripción de recursos que permitan sistemas de recuperación más inteligentes.

(16) http://www.google.com/search?q=Directed+Acyclic+Graph (2007-10-25). Con esa metodología se han desarrollado herramientas para la creación y gestión de diccionarios monolingües basados en una taxonomía conceptual con una única relación en forma de grafo orientado acíclico, donde una categoría puede tener más de un padre. Es este un paso más en el camino que se está recorriendo hasta llegar a herramientas que gestionen bases de datos léxicas bien estructuradas y con múltiples relaciones semánticas (Vaquero, Álvarez y Sáez, 2005).

(17) http://aufderheide.info/lexmlwiki/index.php?title=Legal-RDF:Extent (2008-07-18).

(18) Vid. COM (2003) 406 final. Propuesta de decisión del Parlamento Europeo y del Consejo relativa a la prestación interoperable de servicios paneuropeos de administración electrónica al sector público, las empresas y los ciudadanos (IDABC), p. 24.

(19) http://ec.europa.eu/information_society/eeurope/i2010/index_en.htm (2008-07-18).

(20) Seguridad Social, reconocimiento de pensiones, asistencia sanitaria, permisos de residencia, empleo, estudios, reconocimiento de cualificaciones profesionales.

(21) Registro de nuevas empresas, devolución del IVA, contratación pública a nivel paneuropeo.

(22) http://ec.europa.eu/idabc/en/document/2097/5644 (2008-07-18).

(23) http://www.060.es/guia_del_estado/programas_de_la_administracion/administracion_electronica/LAECSP_11_2007/index-ides-idweb.html (2008-07-18).

(24) Este proyecto se encuentra participado por la Unión Europea a través del proyecto IST 2000-28125, y por la Ducht Tax and Customs Administration, O\&I Management Partners, LibRT, University of Amsterdam (NL), Aplication Engineers, Fortis Bank Insurance (B) y Mega Internacional (F).

(25) En él participan la Unión Europea, a través del proyecto IST 2000-28199, Project Automation, Ministry of Justice, CNR (I), Ministry of Justice (POL), Sema Group, S. A. E. (SP), Intrasoft International (L), Universit Paul Sabatier (F) y University of Amsterdam (NL).

(26) Vid. la página www.metalex.eu/wiki/index.php/main_page, donde se está discutiendo actualmente la versión 1.3.1 (2007-10-13).

(27) Debe caracterizarse como una ontología del derecho para la Web Semántica. Se puede definir como Web extendida, dotada de mayor significado, en la que cualquier usuario de Internet podrá encontrar respuestas a sus preguntas de forma más rápida y sencilla gracias a una información mejor definida. Al dotar a la Web de más significado

Scire. $15: 1$ (en.-jun. 2009) 87-110. ISSN 1135-3716. 
y, por lo tanto, de más semántica, se pueden obtener soluciones a problemas habituales en la búsqueda de información gracias a la utilización de una infraestructura común, mediante la cual es posible compartir, procesar y transferir información de forma sencilla (http://www.w3c.es/Divulgacion/Guiasbreves/WebSemantica) (2007-10-24).

(28) Lo que podría resultar perfectamente aplicable a las actas de las sociedades mercantiles que deben inscribirse en el Registro Mercantil.

(29) Definida como una clasificación basada en una parte de la relación (Boer, Winkels y Vitali, 2007).

(30) Véase http://www.leibnizcenter.org/docs/boer/jurix2007-aboer-submitted.pdf (200710-24).

(31) En 2003 se leía una noticia que indicaba que se había creado una «máquina» para escribir leyes. http://bollettinosegretari.interfree.it/legimatica.htm (2007-10-14).

(32) Vid. www.ittig.cnr.it/TrasferimentocConsulenza/index.php (2007-10-14).

(33) Tanto Eur-Lex como el Senado en España utilizan el tesauro Eurovoc, con términos prefijados por los creadores del sistema para realizar las búsquedas.

(34) En nuestro país esta iniciativa se recuperó por los registradores mercantiles españoles incorporándose a la llevada a cabo por XBRL España junto al Banco de España, la Comisión Nacional del Mercado de Valores y empresas relacionadas e interesadas en este nuevo estándar internacional.

(35) En Alemania, el Registro Mercantil indica la importancia de utilizar el formato XML destacando la necesidad de elaborar una DTD específica para la publicación del Boletín Oficial. Vid. Projectinformation. Bekantmachungen im elektronischen Bundesanzeiger, de 12 de junio de 2003, en www.ebundesanzeiger.de (2007-10-14).

(36) Además de las entidades asociadas a este foro existen diversos organismos que han contribuido al desarrollo de la especificación, como el propio grupo crXML, el Registro Mercantil de Irlanda, el Ministerio de Finanzas checo, la Cámara de Comercio holandesa, la Oficina de Registro y Patentes sueca y el Registro Mercantil de Reino Unido.

(37) El European Business Register es una red para el intercambio de información basada en la interconexión del SBS (Service Broker System). El Registro Mercantil Europeo se encarga de facilitar la información oficial sobre las empresas europeas on-line, removiendo las diferentes barreras en cuanto a idioma, tecnología, legislación, etcétera. Este objetivo lo realiza a través de dos proyectos financiados por la Unión Europea, el European Business Register - Open Network (IST-2001-32260) y el European Business Register - Trust and Internet Confidence Service (C27262). La calidad de la información está asegurada con la participación de los registros oficiales nacionales de cada Estado miembro.

(38) El NDR, junto con el Registro Mercantil Central noruego, está desarrollando una plataforma para el intercambio de información a través de Internet en el seno del proyecto Framework for Internet-based Registration and Filing Project (FINER). Vid. http://www.nrd.no/Webdesk/netblast/pages/index.html?id=28651 (2007-10-14). El NDR se constituyó en 1995 y está integrado por la Fundación Estatal de Desarrollo de Noruega, por Foinco Invest y por sus empleados.

Scire. $15: 1$ (en.-jun. 2009) 87-110. ISSN 1135-3716. 
(39) El European Commerce Registers Forum fue constituido en Cardiff en el año 2000 con la participación de 21 países. Su comité directivo está integrado por representantes de Italia, Reino Unido, Noruega y Suecia, y lo dirige Irlanda (Information Technology Committe). Los principales objetivos del foro son el desarrollo eficiente de los registros en cuanto a la innovación tecnológica en los negocios, especialmente en el seno del SME. Además se apoya a los Estados asociados, especialmente del centro y este de Europa, en la preparación para su integración en el mercado de la Unión Europea, la apertura y desarrollo de medidas de transparencia para las empresas en Europa y la simplificación del desarrollo reglamentario con el fin de reducir los trámites administrativos sobre las empresas.

(40) Vid. algunos estudios empíricos en los que se demuestra la relevancia de la divulgación de esta información desde el punto de vista económico: Bonsón y Gandía (2002), Gandía (2002, 2003a, 2003b y 2003c), Bonsón, Escobar y Gandía (2004), Gandía y Andrés (2005).

(41) Normas Internacionales de Contabilidad (IAS) y Normas Internacionales de Reporting Financiero (IFRS).

(42) Vid. La Gaceta de los Negocios, 10 de febrero de 2005, p. 48.

(43) Ibídem.

(44) Toda la información se puede consultar en https://www.registradores.org/mercantil/ alta.jsp (2008-03-26).

(45) El 11 de junio de 2003, la Comisión aprobó la Directiva del Parlamento Europeo y del Consejo por la que se modifica la Directiva 68/151/CEE del Consejo (Primera Directiva de Sociedades) en lo relativo a los requisitos de información con respecto a determinados tipos de empresas.

\section{Referencias}

Arellano, C.; Nogales, J. T.; Martín, B. (2003). La organización hipertextual del ordenamiento jurídico. Posibilidades y estándares relacionados. // Revista General de Información y Documentación. 2 (2003) 181-191.

Avantic. www.avantic.es (2007-10-24).

Boer, A. (2007). What's in a interchange standard for legislative XML? // Quaderni - Centro Nazionale per l'Informatica nella Publica Amministrazione (anno II - novembre). http://www.one-lex.eu/Activities/summerschool/materials/winkels/Interchange Standard.pdf (2007-10-14).

Boer, A.;Winkels, R.; Vitali, F. (2007). XML standards for law: MetaLex and LKIF. // Jurix 2007. http://www.leibnizcenter.org/docs/boer/jurix2007-aboer-submitted.pdf (2007-10-24).

Boer, A.; Winkels, R. (2007). Standardisation of legal knowledge. // Legislative XML Summer School (2007). http://www.one-lex.eu/Activities/summerschool/materials/boer/ Standardisation\%20Legal\%20Knowledge.pdf (2007-10-24).

Bonsón, E.; Coffin, A.; Watson, L. (2002). XBRL: un lenguaje para el reporting digital. // Partida Doble. 117 (diciembre, 2002) 16-22.

Bonsón Ponte, E.; Gandía Cabedo, J. L. (2002). El código de conducta de AECA. Recomendaciones para la divulgación financiera en Internet. // Revista de AECA. 61 (2002) 3-7.

Scire. $15: 1$ (en.-jun. 2009) 87-110. ISSN 1135-3716. 
Bonsón, E.; Andrés, J. de (2003). XBRL: un estándar para el intercambio electrónico de información. Madrid: AECA ("Monografías", 2) 2003.

Bonsón Ponte, E.; Escobar Rodríguez, T.; Gandía Cabedo, J. L. (2004). Del Código de Buenas Prácticas de AECA a la Ley de Transparencia. // AECA: Revista de la Asociación Española de Contabilidad y Administración de Empresas. 66 (2004) 46-50.

Breuker, J.; Winkels, R. (2007). Use and reuse of legal ontologies in knowledge engineering and information management. // ICAIL (2003). http://www.lri.jur.uva.nl/ $\sim$ winkels/LegOnt2003/Breuker.pdf (2007-10-13).

crXML.org (2001). crXML DRAFT specification, versión 0.9, 2001.

Dick, K. (2000). XML A manager's guide. Nueva York: Addison Wesley, 2000. 165 y ss.

European Business Register XML. www.briteproject.es (2007-10-14).

ESTRELLA: European Project for Standardized Transparent Representations in order to Extend legal Accessibility. http://www.estrellaproject.org (2007-10-13).

Gandía Cabedo, J. L. (2002). La divulgación de información sobre intangibles en Internet: evidencia internacional. // Revista Española de Financiación y Contabilidad. XXXI:113 (2002) 767-802.

Gandía Cabedo, J. L. (2003a). Intangibles disclosure information on Internet by multinational corporations. // The International Journal of Digital Accounting Research. 3 (2003) 61-100.

Gandía Cabedo, J. L. (2003b). La regulación de la información financiera digital: presente y futuro. // Revista de AECA. 64 (2003) 61-63.

Gandía Cabedo, J. L. (2003c). E-gobierno corporativo y transparencia informativa en las sociedades cotizadas. // Revista de AECA. 62 (2003) 3-6.

Gandía Cabedo, J. L.; Andrés Pérez, T. (2005). E-Gobierno Corporativo y transparencia informativa en las sociedades cotizadas españolas: un estudio empírico. Madrid: CNMV ("Monografías", 8), 2005.

GJXDM. http://www.it.ojp.gov/topic.jsp?topic_id=43 (2008-02-22).

Legal-RDF. http://www.w3.org/RDF (2008-07-12).

LEXML. http://www.lexml.de (2008-03-26).

LEXML Italia. www.lexml.it (2008-03-26).

Magnusson Sjöberg, C. (2002). XML as a tool for legal validity in a security context. // XML Europe 2002 (Barcelona, 20 a 23 de mayo de 2002).

MetaLex. http://wiki.metalex.eu/index.php/Workshop_Agreement (2008-07-18).

Milani, P.; Mondorf, A. (2007). Process Ontology for a collaboration framework among Business Registers in Europe. // The e-Government Interoperability conference (10 de octubre de 2007). http://www.briteproject.net/files/brite_paper_parisfinal.pdf (2007-10-25).

Muller, M. (2002). Legal RDF dictionary. // XML Europe 2002 (Barcelona, 20 a 23 de mayo de 2002). http://www.lexml.de/legal_rdf_dictionary_Barcelona.htm (2007-10-24).

TESTA: Trans European Services for Telematics between Administrations.

Tiscornia, D. (2007). Structuring multilingual ontologies for law. // Ajani, G.; Peruginelli, G.; Sartor, G.; Tiscornia, D. (eds.) (2007). The Multilanguage Complexity of European Law: Methodologies in Comparison. Florencia: European Press Academic Publishing,

Scire. $15: 1$ (en.-jun. 2009) 87-110. ISSN 1135-3716. 
2007. 145-162. http://www.one-lex.eu/Activities/summerschool/materials/tiscornia/ Tiscornia.pdf (2007-10-24).

Vañó Vañó, M. ${ }^{a}$ J. (2004). Información y gobierno electrónico en las sociedades cotizadas españolas. // RDBB. 95 (julio-septiembre 2004) 77 y ss.

Vañó Vañó, M. ${ }^{a}$ J. (2005). XML, una herramienta al servicio del buen gobierno corporativo. // Revista Aranzadi de Derecho y Nuevas Tecnologías. 8 (2005) 101-116.

Vaquero Sánchez, A.; Álvarez Montero, F.; Sáenz Pérez, F. (2005). Diccionarios basados en taxonomías con estructuras de grafo orientado acíclico. // Procesamiento Del Lenguaje Natural. 35 (2005) 259-266. http://www.sepln.org/revistaSEPLN/revista/35/32.pdf (2007-10-24).

XBRL. http://www.xbrl.org (2007-10-24).

Your Europe. http://ec.europa.eu/youreurope/(2007-10-24).

Recibido: 2008-03-26. Aceptado: 2008-10-01

Scire. 15 : 1 (en.-jun. 2009) 87-110. ISSN 1135-3716. 